\title{
AN APPLICATION FOR CULTURAL HERITAGE IN ERASMUS PLACEMENT. SURVEYS AND 3D CATALOGUING ARCHAEOLOGICAL FINDS IN MÉRIDA (SPAIN)
}

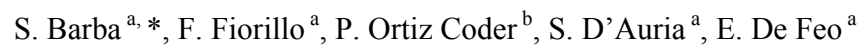 \\ ${ }^{a}$ UNISA, Department of Civil Engineering, 84084 Salerno, Italy - (sbarba, ffiorillo)@unisa.it \\ ${ }^{\mathrm{b}}$ GAVLE, 06800 Mérida (Badajoz), Spain - pedro@gavle.es
}

KEY WORDS: Vivid 910 and Geomagic Studio, Texture, 3D reconstruction, PDF visualization

\begin{abstract}
:
Man has always had the need to live with his past, with its places and its artefacts. The reconstructions, the economical changes, the urbanization and its speculations have devastated whole cities, changed the faces of their historical centers, changed the relationship between the new and the old. Also the millenarian 'rest' of the archaeological findings, and therefore the respect towards those ancient civilizations, has been troubled.

Our continent is rich in masterpieces that the modern man are not able to protect and pass on to the future, it is commonplace to observe that the modern 'civilization' has cemented and suffocated the ancient city of Pompeii, or even worse, failed to protected it. Walking in the archaeological area of Paestum it can be noticed how just sixty years ago, no one had the slightest concern of fencing the amphitheatre and the Roman forum, or entire houses and shops, to lay a carpet of tar or simple to build constructions completely inferior compared to those majestic Greek temples.

The engineers and the architects should be held responsible for this as based on their scientific and humanistic sensibility; they should bring together the man with his surroundings in the complete respects of the historical heritage.

The interest in ancient began to change nearly three decades ago since it was realized that the "Cultural Heritage" is a major tourist attraction and, if properly managed and used, it can be an economical cornerstone. Today, thanks to survey and the 3D graphics, which provide powerful new tools, we are witnessing a new and real need for the conservation, cataloguing and enhancement as a way to revive our archaeological sites.

As part of a major laboratory project, artefacts from the Roman period (I and II century b.C.), found in the Spanish city of Mérida, declared World Heritage by UNESCO in 1993, were acquired with a 3D laser scanner VIVID 910, and then catalogued.

Based on these brief comments we wanted to direct the work carried out under an Erasmus Placement Agreement with the Spanish company Gavle, towards the info-graphics detection and documentation of this archaeological heritage.
\end{abstract}

\section{INTRODUCTION}

The work is about 3D laser scanner survey of archaeological findings (dated back to I-II century b.C.) made by Consorcio de la Ciudad Monumental, Histórico-Artística y Arqueológica de Mérida and the subsequent creation of photo-realistic 3D models. The area of the findings interests the archaeological zone of Mérida, a spanish city in Extremadura, become a Unesco World Heritage in 1993. The project was inspired by a collaboration with GAVLE, a spanish graphic heritage surveying company, within Erasmus Placement program. The traditional method of cataloguing the findings (e.g. jewels, vases, lamps etc. as can be seen in Figure 1), capturing their shapes and defining their colorimetric aspects requires long and inconvenient times. Traditional methods are still used for its cheapness and facility but the final output are very inaccurate and hard to understand by any other but archaeologists itself.

The aim of this work was to use the laser scanner technology and the latest computer graphic resources in the archaeological field (Guidi, 2010), too often still outdated, to speed up data acquisition and to create an innovative cataloguing method (both paper and computer).

This method allows the experts to have the objects models at their disposal at any time preventing the valuable archaeological remains from wearing due to repeated handling. The model is a 3D photo-realistic representation, so realistic to permit the realization of a hyper-realistic virtual museum to view, study and analyze the archaeological remains (Qingquan et al., 2005).
There are different 3D capture procedure, depending on the purpose. A virtual representation of the object that meets its real volumetric shape and its texture will be sufficient for an overall view (Iannizzaro et al., 2009).

Instead, a dimensional inspection requires a perfect match with the original geometry and a greater attention for the details.

In this specific project the final models are corresponding with the real ones both in the dimensions (accurate to a tenth of a millimeter) and in the accuracy of the details of the surfaces, included the colorimetric aspect.

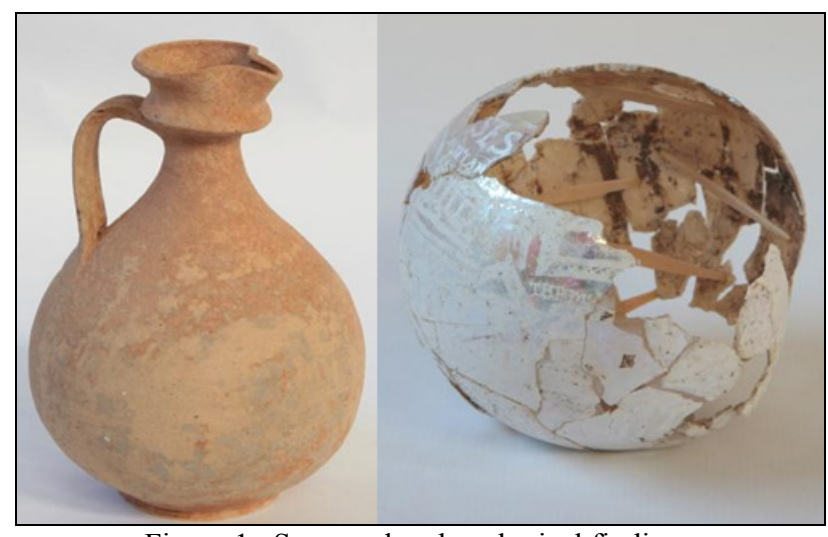

Figure 1. Surveyed archaeological findings

* Corresponding author. 


\section{DETAILED SURVEYING INSTRUMENTS}

The choice of data capture system depends on the aim and on the features of the object we want to survey (Barba, 2008). In our case, most of the object to be surveyed were small in size (it ranged from small items such as a teaspoon to medium volume elements such as a flower pot); only few of them measure more than 1 meter in one of the different dimensions. In addiction 3D models were aimed at science-teaching use so was essential to achieve a high degree of accuracy and resolution (Abate et al., 2009). For these particular reasons the laser triangulation scanner, non-contact 3D digitizer VIVID 910 by Konica Minolta was chosen (Figure 2).

This device allows to obtain three-dimensional models in the triangular mesh with great accuracy in a relatively short time (Barba, 2008). The instrument is equipped with three different focal distance as each case requires: tele $(\mathrm{f}=25,5 \mathrm{~mm})$, middle $(\mathrm{f}=14,5 \mathrm{~mm})$ and wide $(\mathrm{f}=8 \mathrm{~mm})$. For most of the findings surveyed (in all 46) we used a tele lens for its longer focal length and the possibility of a greater focus on the object that fulfill our need of having a model as accurate as possible. The number of scans for each finding, from a minimum of 4 to a maximum of 12, was determined by the need to obtain an accurate model of the surfaces with no significant information gaps, the so called holes, in the mesh. So that the resulting model is not a fake, doesn't have shadow area or processing errors and can be used for research purposes. For small objects it also can be used a rotating stage set that allows the object to be surveyed from different perspectives. It consists in a rotation of the object on a vertical axis while the equipment is maintained fixed. These supporting instruments simplifies the data capturing stages and its post-processing (Mara et al., 2007). The right calibration of the rotating table involves little control by the operator and returns an excellent 3D model. But we didn't use it for several reasons: the archaeological findings were too precious and fragile to be undergone at such automatic movement and their shapes were hardly ascribable to standard solids of revolution. The scans were made defining every time both the distance (from 60 to $120 \mathrm{~cm}$ ), between the finding and the VIVID 910, and the perspectives depending on the features and the size of the objects. The software used to control the laser scanner during the surveying step was Polygon Editing Tool (PET), provided by the same producer of the laser. Autofocus and fine mode scan quality were chosen. A fine mode scan quality involves the laser beam scans three times the surface of the object so as to have a resolution of a tenth of millimeter.

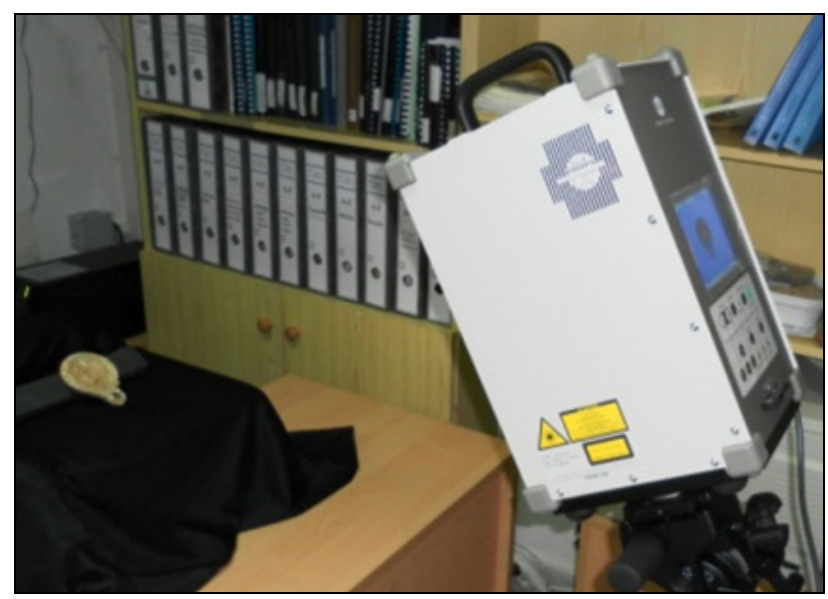

Figure 2. Data acquisition with the laser scanner VIVID 910

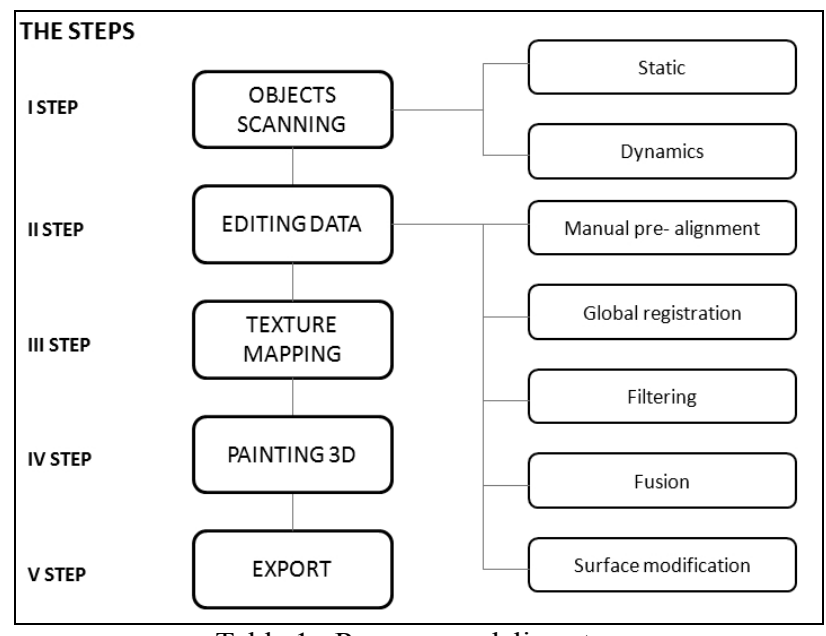

Table 1: Reverse modeling step

With PET it's also possible to manage captured data and postprocessing them, but we preferred, for reasons we will explain later, to use another software of reverse engineering: Geomagic Studio because it offers a plenty of utilities and a user-friendly interface.

Moreover it offers several popular output file format increasing the portability between different photo-retouch, computer graphics, reverse engineering and 3D models management software. So also freeware software users are able to read these files.

\section{REVERSE MODELING STEP (I-II STEP)}

The main steps of the work of $3 \mathrm{~d}$ models creation (Table 1) were five.

Having completed the scans from different perspectives was then important to align the resulting point clouds, generated by Polygon Editing Tool. To do this it's necessary to identify at least three homologous points between every different scan (Figure 3).

In order to do this it's important to have an overlay surface, at least equal to $30 \%$ of the scanned part (Barba, 2008). In this way much of the post-processing work was done easily.

Polygon Editing Tool allows to manage not only point clouds but also mesh surface but for this we prefer to use Geomagic Studio for its superior capabilities in handle the texture mapping; 3D models can be generated starting from point clouds or from mesh surfaces.

The first method was used when the 3D model captured needed a noise reduction and the second one when we already had a 3D model with a fine info-graphic aspect and a regular size.

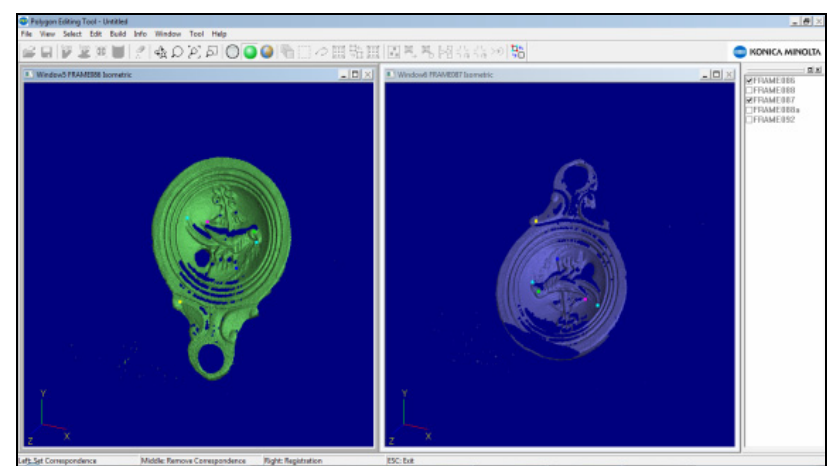

Figure 3. Manual registration in Polygon Editing Tool 
Another important step in registration was the elimination of the scanned part which were irrelevant to the archaeological finding.

First because we would have a too large file and second because noise data can invalidate a perfect registration and this will involve the operator in an awful lot of work in the post processing step. Obviously all this process will not weaken the size reliability of the model (Guidi, 2010).

To define the complete volume of the object was used the global registration algorithm iterated till when it gives a value of standard deviation accepted by the operator (Figure 4). For example with the model of a lamp we accepted a value less than $0,108 \mathrm{~mm}$.

Then we were able to merge all the different scans in one single model so as to have the real texture. This was possible because during the scans the survey beam, thanks to an integrated CCD sensor, captured a series of two-dimensional images. The sensor however was made up of only 2 megapixel so was necessary a step of the so-called painting $3 \mathrm{D}$ to apply professional shots on 3D models.

Possible gaps such as shadow area or data acquisition error due to the reflection of the material were fixed with the "fill holes" algorithm. With small holes was used the automatic operational mode, while the manual operational mode was used with larger holes.

After the filling new different surfaces were generated: some followed the curvature of the edge other the tangents of the surrounding mesh and other were flat.

With other algorithms was possible to reduce the number of polygons so as to obtain a more smooth surface.

This step requires particular attention in order to obtain very realistic models and to avoid a mere reproduction or even worse a false.

\section{PHOTO-REALISTIC MODELS: TEXTURE MAPPING AND PAINTING 3D (III-IV STEP)}

\subsection{Texture mapping}

The aim of the work was to obtain a photo-realistic 3D model, that is to say an object with exactly the same graphic of the original. To do this we proceed with the additional texture mapping step.

At the moment of the scanning the correspondence between the point of the model and its colour is not very good due to the low resolution of the CCD sensor and the condition of lighting. So the process of texturing allows us to add detailed information about the materials and some other formal details on the digital model without complicate geometric complexity of it. This result was obtained projecting on the object images of the texture.

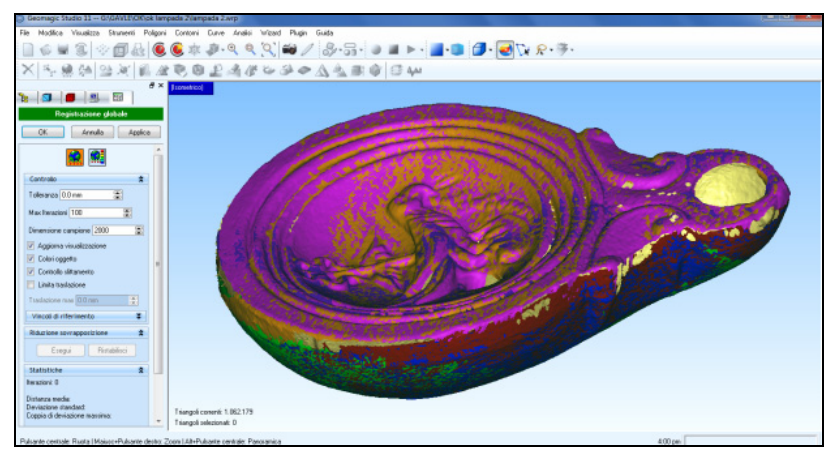

Figure 4. Global registration in Geomagic Studio

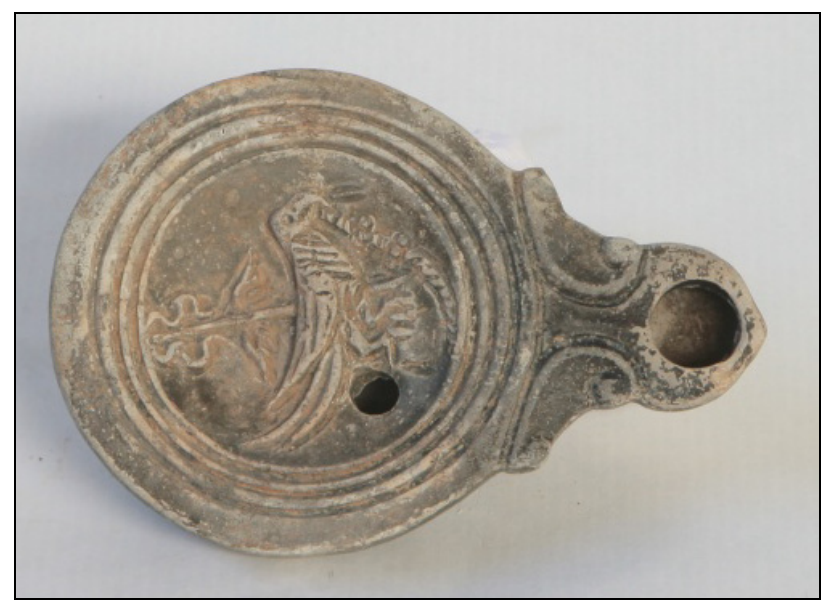

Figure 5. Image of a roman lamp for the photo texturing

This step is peculiar because it treats the matching of opposite data acquisition, in our case those from a laser scanner and those from a digital camera that are different in setting, time and many other things. They are difficult to match because the point acquired by the laser and the pixels captured by the camera lack a one-to-one correspondence (Girardi et al., 2007). The texture is an image so $3 \mathrm{D}$ measuring instruments were the same used for static shots: the camera. We used a Canon EOS $400 \mathrm{D}$ with a resolution of 10 megapixel.

Before starting the photographic survey we have to make sure of having a perfect setting (Ericsson et al., 2007), similar to the condition of a photographic studio; because obtain neutral lights and avoid shades and light reflections means simplify the work in the photo-processing step (Figure 5).

For these reasons we used a white background, an indirect spotlight illumination and the framing parallel to the object plane. Then, having completed the snapshots, the correspondence between the point clouds and the digital images was realized by hand. At first we continued using Geomagic Studio with which is also possible processing raster images, allowing the simultaneously loading of both $3 \mathrm{D}$ model and the corresponding photo (Figure 6), previously processed with a photo retouching software like Photoshop (in order to isolate the background from the surface to spread on the model).

Once we individuated at least 15 homologous points we proceed with tools like generate texture, project image and manage texture in spreading the texture on the model.

Homologous point were situated mostly near the holes or particular sections in relief or micro-fractures.

Obviously the more the model is detailed the more homologous point have to be chosen near the point of interest, avoiding image deformation when applying the texture. Photo-realistic model is not yet completed, another step is needed.

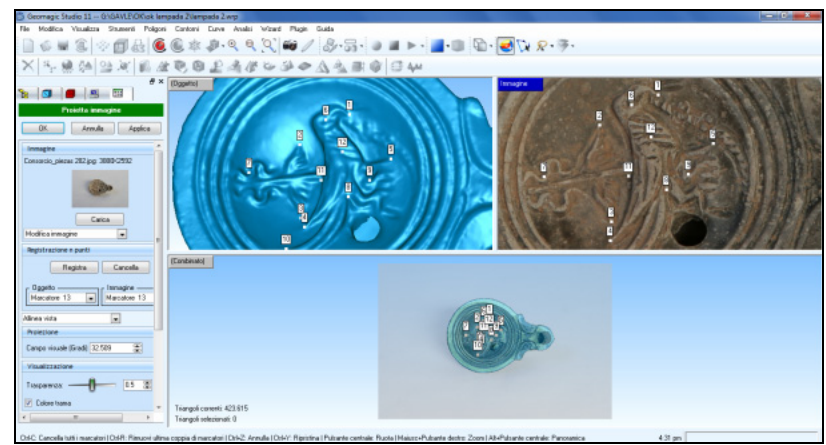

Figure 6. Texture mapping in Geomagic Studio 


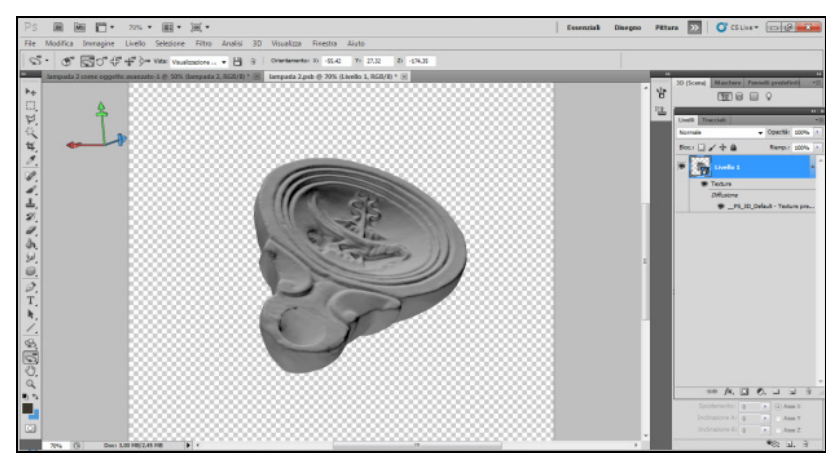

Figure 7. Painting 3D in Adobe Photoshop CS5 Extended

\subsection{Painting 3D}

Working only with Geomagic Studio was not possible to avoid a discontinuity at the boundaries of the different projected images. For this reason we chose a 3D painting software which is very useful for specific digital editing; in fact, even if it's not very suitable to project real texture on $3 \mathrm{D}$ geometrically defined objects, it's designed to change items already textured (Girardi et al., 2007). An interesting result was obtained using Adobe Photoshop CS5Extended. The program allows you to paint and modify the color information by working directly on the $3 \mathrm{D}$ model and using the same tools used for two-dimensional objects such as Clone Stamp, Brush, Smudge, etc.

The Extended version is in fact able to read 3D models already textured with Geomagic Studio. It in fact manage them as if the models were simple two-dimensional images (Figure 7). The result obtained is very satisfactory (Figure 8). It opens new horizons in the field of survey with the purpose of archaeological cataloguing.

\section{EXPORTING FILE FORMAT (V STEP)}

Using multiple software at once and, above all, try to maintain high the quality reducing file size is a very relevant issue. For these reasons was required a specific study on the interchange formats that allowed us to keep tracks of all types of format used in the various passage from one software to another. In the object scanning step the extension of the output file of the laser scanner software was CMD.

Such a file was used to edit the initial point cloud but then, to export file in Geomagic Studio, was better change it in VVD (a format owned by Konica Minolta). This was done in order to convert automatically the point cloud in a polygonal surface and to make it easier to treat. Geomagic Studio can save models in various file format.

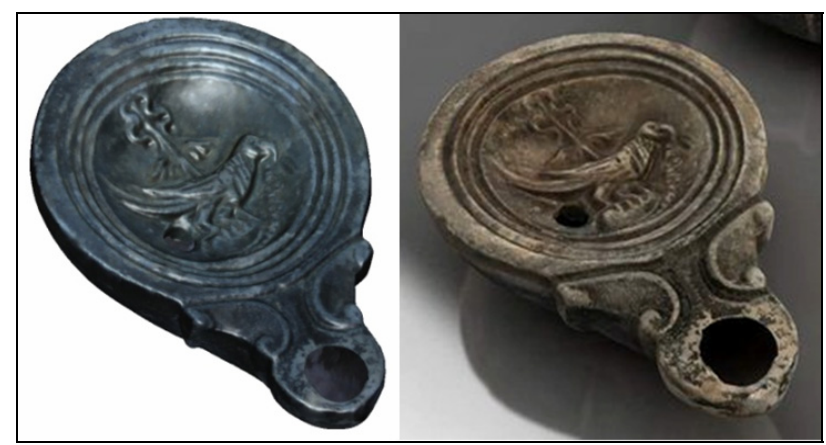

Figure 8. 3D model with automatic and photo-realistic texture

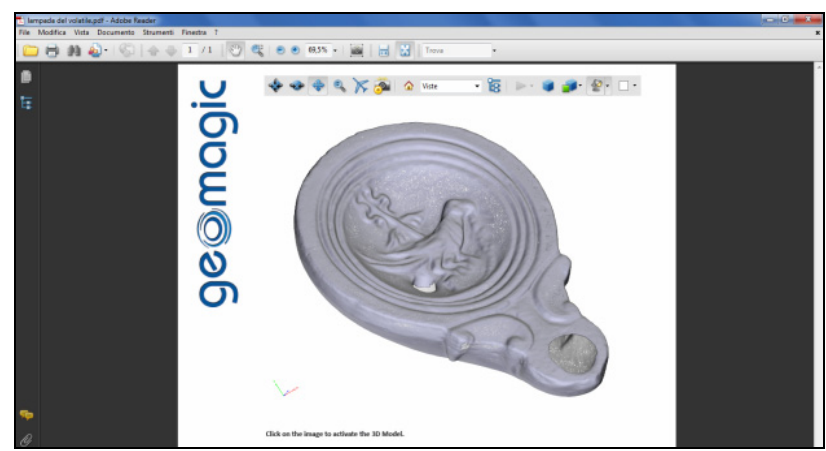

Figure 9. 3D model in PDF3D file format

The most commonly used and suitable to our needs were WRP, STL, VRML, OBJ.

WRP is the file format assigned by default and it is compatible with many software of reverse engineering but it not always automatically recognize the texture.

The STL file format - Standard Triangulation Language - keeps only the information about the geometry of the digital model and can be imported into most modeling software and also into numerical control machine managing software.

The VRML - Virtual Reality Modeling Language - is designed for multimedia applications and for 3D interactive representation on Internet. With this file extension is also possible to associate with the model some polygonal features such as texture, brightness etc.

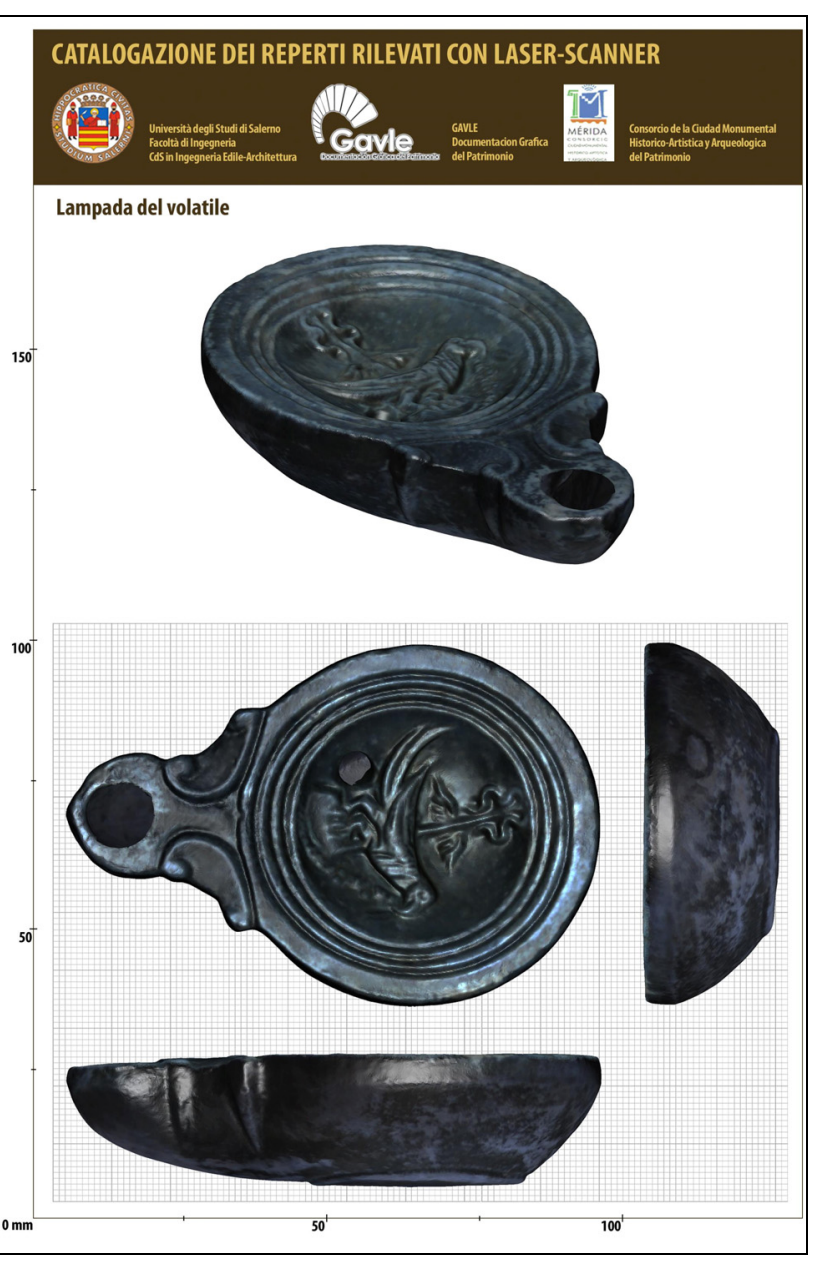

Figure 10. Cataloguing sheet 


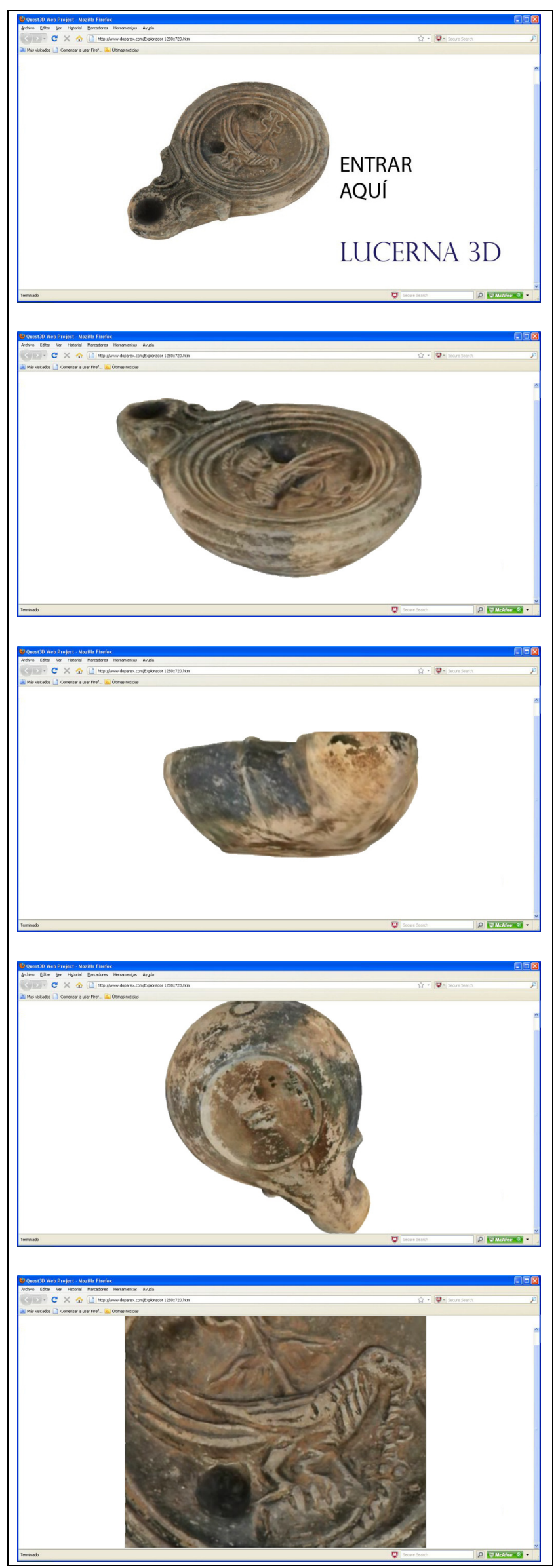

Figure 11. Video frame
OBJ - Object file - allows you exporting of the geometry and the texture eventually associated with the model and lets you import polygon in some particular modeling software. Moreover, OBJ is the only file extension AdobePhotoshop CS5 Extended is able to recognize, allowing photo-retouch directly on the 3D model. In fact, when you export a model in OBJ, Geomagic Studio creates some supporting image files (in JPEG or PNG) and in this way the graphics editing program can directly edit the image file corresponding to the portion of the surface of the 3D model we are editing.

The extensions of the files mentioned above are primarily used by expert. But there are also more common formats, suitable for anyone else. They include, for example, VAT, with which you can make movies of the model while moving, or the recent PDF3D (Figure 9) that allows you to easily manage point cloud or mesh in an intuitive and extremely easy way. The latter format allows the visualization of the 3D model just having AdobeReader and also allows you to interact with the 3D model in an intuitive way by making linear and volumetric measurements or crossing section with generic plans or by default. It's also possible to change the style of visualization (point cloud, wire-frame, mesh, etc.).

This is a revolutionary solution because we can sum up at the same time, in the same file format, videos, photos and text and obviously our 3D model representation (Figure 10).

Another solution for web representation can be a 'video' realized using a particular software (Figure 11), so anyone with a computer can see the $3 \mathrm{D}$ model of an archeological remain downloading a simple plug-in. At this link an example of it: http://gavle.es/site/2010/11/lucerna-un-modelo-3d-perfecto-yonline (the plug-in used is Quest3D); this work was supported by Almudena Torres y Carlos Acevedo.

\section{CONCLUSIONS}

The most innovative goal of the work is linked mainly to the step of 3D painting that gave responses to the demands of realism of the 3D models (Abdelhafiz et al., 2005). The process described can be extended to a wide series of applications: monitoring the conservation status of the ornaments, the computer cataloguing of precious objects preserved in museums, the creation of a virtual museum accessible remotely by Internet. It can be a revolution for scholars who are unable to reach the archaeological sites or simply for whom is interested in the objects such as industrialists, art lovers and curious.

This innovation will help from a loads of point of view: archaeological findings will no more undergo stress due to continuous handling and will represent a great leap forward for e-learning and last but not least will put in new light Archaeology discipline.

\section{REFERENCES}

Abate D., Baracca M., Ciavarella R., Furini G., Migliori S., Pierattini S., 2009. Laser scanner technologies and 3D models integration: analysis and methodological approach. Workshop finale dei progetti PON ricerca 2000-2006, Catania, Italy.

Abdelhafiz A., Niemeier W., Riedel B., 2005. Report on the Joint ISPRS Commission V/IV Workshop "Virtual Reconstruction and Visualization of Complex Architectures", Mestre-Venice, Italy. http://www.isprs.org/proceedings/XXXVI/5-W17/pdf/31.pdf 
Barba S., 2008. Tecniche digitali per il rilievo di contatto. CUES, Salerno.

Ericsson M., Hast A., Reiner T., 2009. Report on the Joint ISPRS Commission V/IV Workshop "3D Virtual Reconstruction and Visualization of Complex Architectures", Trento, Italy.

http://www.isprs.org/proceedings/XXXVIII/5-W1/pdf/hast_etal.pdf

Guidi G., Russo M., Beraldin J., 2010. Acquisizione 3D e modellazione poligonale. McGraw-Hill, Milano.

Girardi S., Gonzo L., Pontin Marco, Remondino F., Rizzi A., Voltini F., 2007. Integrazione di fotogrammetria e laser scanner per la documentazione di Beni Culturali. In: XI ASITA Conference, Torino, Italy.

Mara H., Niccolucci F., Kampel M., Sablatnig R., 2007. Report on the Joint ISPRS Commission V/IV-I Workshop "3D Virtual Reconstruction and Visualization of Complex Architectures", ETH Zurich, Switzerland.

http://www.isprs.org/proceedings/XXXVI/5-W47/pdf/mara_etal.pdf

Qingquan L., Yuguangm L., Zhi W., 2005. Report on the Joint ISPRS Commission V/IV-I Workshop "Virtual Reconstruction and Visualization of Complex Architectures", Mestre-Venice, Italy.

http://www.isprs.org/proceedings/XXXVI/5-W17/pdf/20.pdf

Iannizaro V., Barba S., Giordano M., 2009. Nuvole di punti vs Nuvole di pixel. In: $7^{\text {th }}$ International Forum "Le vie dei mercanti", Capri, Italy.

\section{ACKNOWLEDGEMENTS}

Special thanks to Consorcio de la Ciudad Monumental, Histórico-Artística y Arqueológica de Mérida. 